\title{
Comparison of cone-beam computed tomography and digital panoramic radiography for detecting peri-implant alveolar bone changes using trabecular micro-structure analysis
}

\author{
Guldane Magat ${ }^{1}$, Elif Oncu ${ }^{2, \star}$, Sevgi Ozcan ${ }^{1, \dagger}$, Kaan Orhan $^{3}$ \\ ${ }^{I}$ Department of Oral and Maxillofacial Radiology, ${ }^{2}$ Department of Periodontology, Faculty of Dentistry, Necmettin Erbakan University, Konya, \\ ${ }^{3}$ Department of Oral and Maxillofacial Radiology, Faculty of Dentistry, Ankara University, Ankara, Turkey
}

\begin{abstract}
J Korean Assoc Oral Maxillofac Surg 2022;48:41-49)
Objectives: We compared changes in fractal dimension (FD) and grayscale value (GSV) of peri-implant alveolar bone on digital panoramic radiography (DPR) and cone-beam computed tomography (CBCT) immediately after implant surgery and 12 months postoperative.

Materials and Methods: In this retrospective study, 16 patients who received posterior mandibular area dental implants with CBCT scans taken about 2 weeks after implantation and one year after implantation were analyzed. A region of interest was selected for each patient. FDs and GSVs were evaluated immediately after implant surgery and at 12-month follow-up to examine the functional loading of the implants.

Results: There were no significant differences between DPR and CBCT measurements of FD values $(P>0.05)$. No significant differences were observed between FD values and GSVs calculated after implant surgery and at the 12-month follow-up $(P>0.05)$. GSVs were not correlated with FD values $(P>0.05)$.

Conclusion: The DPR and reconstructed panoramic CBCT images exhibit similar image quality for the assessment of FD. There were no changes in FD values or GSVs of the peri-implant trabecular bone structure at the 12-month postoperative evaluation of the functional loading of the implant in comparison to values immediately after implantation. GSVs representing bone mass do not align with FD values that predict bone microstructural parameters. Therefore, GSVs and FDs should be considered different parameters for assessing bone quality.
\end{abstract}

Key words: Fractals, Bone density, Panoramic radiography, Cone-beam computed tomography, Dental implants

[paper submitted 2021. 8. 2 / revised 2021. 9. 13 / accepted 2021. 9. 28]

\section{Introduction}

Dental implants are a solid, fixed choice for the treatment of total and partial edentulism with high success rates both in primitive and recovered bone ${ }^{1,2}$. The use of dental implants in oral restoration has turned into a standard of interest in daily

\section{Guldane Magat}

Department of Oral and Maxillofacial Radiology, Faculty of Dentistry, Necmettin Erbakan University, Yunus Emre Boulevard, Baglarbast Street, 42090 Konya, Turkey

TEL: +90-505-945 6157

E-mail:gul_dent@hotmail.com

ORCID: https://orcid.org/0000-0003-4418-174X

*Current affiliation: Department of Periodontology, Faculty of Dentistry, Lokman Hekim University, Ankara, Turkey

${ }^{+}$Current affiliation: Department of Oral and Maxillofacial Radiology, Faculty of Dentistry, Nigde Omer Halisdemir University, Nigde, Turkey

(c) This is an open-access article distributed under the terms of the Creative Commons Attribution Non-Commercial License (http://creativecommons.org/ licenses/by-nc/4.0/), which permits unrestricted non-commercial use, distribution, and reproduction in any medium, provided the original work is properly cited.

Copyright (C) 2022 The Korean Association of Oral and Maxillofacial Surgeons. practice $^{3}$.

Primary and secondary implant stability is essential for effective osseointegration of dental implants. Stability depends on the quality and amount of bone, the surgical protocol, and the design of the implant ${ }^{4}$. In the weeks after the placement of the implant, primary stability becomes secondary stability due to bone development and reconstruction at the boneto-implant interface. An essential element for long-lasting osseointegration of an implant-supported prosthesis is the counteractive actions of biomechanical intricacies, which rely on the loads on the outlying bone ${ }^{5}$. Peri-implant bone undergoes a continuous cycle of remodeling that is fundamental to the capacity to maintain bone-to-implant contact (BIC) after loading ${ }^{6}$. Through clinical loading of implants, functional forces, stresses and strains, and prosthesis choice all impact $\mathrm{BIC}^{7}$. The survival rate of dental implants relies on the quality of bone that has been added to expand $\mathrm{BIC}^{8,9}$.

The micro-architecture of the trabecular bone affects im- 
plant stability ${ }^{8}$. Therefore, a density-based structural approach has been preferred. By performing a pre-surgical analysis of the trabecular structure, the outcome of treatment can be predicted and the success rate of the implant increased. In addition, the success of the implant can be monitored by radiographs at regular intervals after implantation.

Recently, studies have shown that fractal dimensions $(\mathrm{FD})^{10}$ are useful for the evaluation of bone quality. Fractal analysis (FA) uses statistical surface examination of the trabecular bone microarchitecture and gives a numerical value for the complexity of an image ${ }^{11,12}$. It is a precise, cautious, and accessible method ${ }^{12-14}$. In dental radiology, FD computation has been used for the identification of bony changes associated with periapical periodontitis, periodontal diseases, bone surgery, and systemic diseases ${ }^{6,10,15-17}$. FD analysis can be used to evaluate surface-related characteristics of BIC and to evaluate trabecular bone structure in radiographs ${ }^{11,18,19}$. Several methods have been proposed for FD calculation, with the box-counting method ${ }^{20}$ being the most often used in dental radiology ${ }^{10}$

As an optimum standard, routine histomorphometry is used to assess bone micro-architecture ${ }^{21}$. However, it is in vitrobased, long-lasting, and costly. Micro-computed tomography $(\mu \mathrm{CT})$ is accepted as a new first-rate method for measuring bone micro-architecture ${ }^{22,23}$, but has not been validated in clinical trials and has only been applied to small animals (in vivo) or biopsy (ex vivo) samples ${ }^{23}$.

$\mathrm{CT}$ is a more accurate method for quantitative evaluation of bone density, and its use has increased over the last 20 years. Previous research demonstrated an association between bone density on CT and primary implant stability ${ }^{24,25}$. However, subjects were exposed to high radiation doses during CT scans. In the late 21st century, the cone-beam CT (CBCT) method was introduced for head and neck imaging was presented. The benefits of CBCT are high-level imaging resolution and the possibility of lower radiation doses and costs than $\mathrm{CT}^{24,26}$. However, CBCT has disadvantages including scattering radiation and bone density values that are not compatible with intensity values. The contrast and resolution of CBCT are also reduced by scatter radiation and artifacts. However, significant correlations were demonstrated between the density level of $\mathrm{CBCT}$ and the Hounsfield unit (HU) of multi-slice CT in recent studies ${ }^{24,26}$. The relationships between CBCT-derived grayscale values (GSVs) and multislice CT-derived GSVs were investigated by Pauwels et al. ${ }^{27}$ who observed questionable outcomes demonstrating a good correlation between $\mathrm{CBCT}$ and $\mathrm{CT}$, as well as wide errors when using GSVs quantitatively. As a result, it is controversial to derive density values from CBCT images. When the sensitivity of CBCT for measuring bone-related parameters (e.g., bone thickness) is thoroughly examined ${ }^{1}$, little information is available about its utility for assessing the structure of peri-implant bone tissue ${ }^{28}$. No research has assessed the relationship between FD and GSV for indicating bone quality.

There have not been sufficient studies evaluating trabecular bone patterns by comparing digital panoramic radiography (DPR) and CBCT. Therefore, the suitability of CBCT for the evaluation of trabecular bone structure should be further researched. In this context, our primary aim was to compare the image qualities of DPR and CBCT using image analysis. We hypothesized that there are no statistically significant differences between CBCT reformatted panoramic images and DPR. Our second aim was to test the applicability of FD in dental implantology. The third aim was to investigate and compare changes in peri-implant bone structure after the functional loading of implants on DPR and CBCT images. The fourth aim was to analyze the relationships between GSV and FD values obtained with DPR and CBCT and bone quality.

\section{Materials and Methods}

This retrospective study was approved by the Local Research Ethics Committee (No. 2018.01) and complied with the guidelines laid out in the Declaration of Helsinki (2013). Sixteen subjects with CBCTs taken about 2 weeks after implantation and one year after implantation were chosen among patients who had dental implants that replaced missing teeth in posterior mandibular sites at the Department of Periodontology, Necmettin Erbakan University Dentistry Faculty, Konya, Turkey, between 2014 and 2017. CBCTs were taken about 2 weeks after implantation to diagnose postoperative complications such as altered sensation. Approximately one year after implant placement, the second set of CBCTs were taken for patients with persistent subjective (not objective) findings such as taste changes, paresthesia, and tenderness in their jaws. The protocol complied with the guidelines of the American Academy of Oral and Maxillofacial Radiology (AAOMR) regarding radiology in dental implantology $y^{29}$. In the first CBCT, the status of implants and anatomical structures were assessed. In the second CBCT, the condition of the implants after prosthodontic loading was re-evaluated. However, no problems were identified with implant operations and prostheses such as mobility or nerve 
damage. Radiographically, there appeared to be no contact of the implant with the mandibular canal. Therefore, our sample was limited to only 16 patients.

\section{Inclusion and exclusion criteria}

Patients with the minimum bone width and height required for placing an implant, which is at least $11 \mathrm{~mm}$ in length and $4 \mathrm{~mm}$ in diameter, without additional bone procedures before placing the implant in the posterior mandibular region were included in the study. Patients who had clinically and radiologically poor implant osteointegration, high-grade bruxism, a background of smoking, intensive alcohol consumption, chemotherapy and radiotherapy, pregnancy, liver pathology, hematologic nephropathy, inflammatory and autoimmune diseases of the oral cavity, and were immune-suppressed and using corticosteroids were excluded from the study. CBCTs of patients with no clinical or radiological problems surrounding the implants were included in the study.

The same surgeon (E.O.) performed all surgical procedures with local infiltration anesthesia (Ultracaine D-S; Hoechst, Abdi Ibrahim, Turkey). A crestal incision was made. After that, a mucoperiosteal flap was elevated. Implant osteotomies were performed, following the recommendations of the Astra Tech Dental Implant System. A total of 60 implants (implant diameters and lengths, $4 \mathrm{~mm} \times 11 \mathrm{~mm}$ ) (OsseoSpeed; Astra Tech, Mölndal, Sweden) were set in adequate mature bone with no fenestration or dehiscence in the posterior mandibular region. Implants were loaded three months after insertion. All prosthodontic rehabilitations were performed by the same specialist. The patients were evaluated every three months for plaque control and oral hygiene.

\section{Radiological evaluation}

All DPR images were obtained by the same digital panoramic machine (Morita Veraviewepocs 3D R100-P; J Morita MFG, Kyoto, Japan) at $70 \mathrm{kVp}, 10 \mathrm{~mA}$, and 10 seconds. The images were then exported as TIFF files. CBCT images were acquired using a Morita 3D Accuitomo 170 (J Morita MFG) operated at $90 \mathrm{kVp}$ and $5 \mathrm{~mA}, 17.5$ seconds rotation time, voxel $0.25 \mathrm{~mm}, 100 \mathrm{~mm}$ field of view. The filtration component was not added. The desired region of each image was located at the center of the CBCT and DPR machines. The occlusal and Frankfurt planes of each patient were positioned parallel to the ground according to the device protocols. The DPR and CBCT images were saved as TIFF files. All radio- logic evaluations were performed by a dento-maxillofacial radiologist (G.M.) with ten years of experience.

\section{Selection of regions of interest and fractal analysis}

FD calculations were evaluated using the ImageJ $1.49 \times$ program (National Institutes of Health [NIH]; http://rsb.info. nih.gov/nih-image). Regions of interest (ROIs) were selected among three regions around each implant in the posterior mandibular area. The ROI was set to a width of 25 pixels and a height of 50 pixels $(25 \times 50$ pixels $)$ at the first macro thread around the mesial and distal parts of every implant on DPRs and CBCT images. However, the ROI was set to a width of 50 pixels and height of 25 pixels $(50 \times 25$ pixels $)$ at the last macro thread around the apical parts of every implant on DPRs and CBCT images since the implant was close to the mandibular canal.(Fig. 1) The ROIs were defined away from the crestal bone, the adjacent tooth roots and lamina dura, and other structural entities. Because remodeling develops throughout the bone that is within a $1 \mathrm{~mm}$ radius from the periphery of the implant ${ }^{7}$, the ROI was set to a width of $2.0 \mathrm{~mm}$ around the radius of the BIC to avoid artifacts created by the implant. Images with artifacts within the ROI field were also excluded from the study.

The FD was measured according to the protocol of White et al. ${ }^{30}$. The ROIs were selected and filtered via Gaussian blur with a sigma value of 3 . The obtained image was then subtracted from the original image and 128 added at each pixel location to distinguish bone marrow spaces and trabeculae

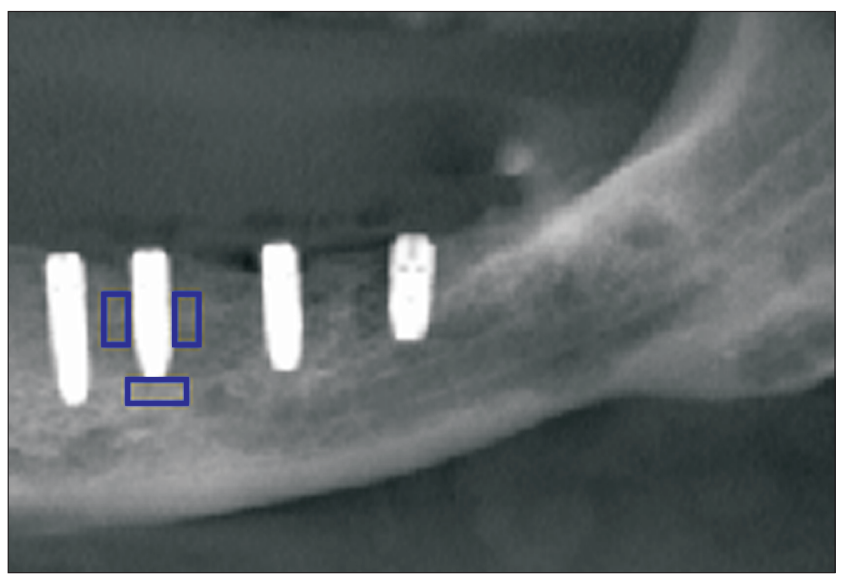

Fig. 1. Region of interest selection at mesial $(25 \times 50$ pixels), distal $(25 \times 50$ pixels), and apical $(50 \times 25$ pixels $)$ areas on digital panoramic radiography and reformatted panoramic images of conebeam computed tomography.

Guldane Magat et al: Comparison of cone-beam computed tomography and digital panoramic radiography for detecting peri-implant alveolar bone changes using trabecular micro-structure analysis. J Korean Assoc Oral Maxillofac Surg 2022 
and the ROI was binarized and outlined. The resulting image was eroded, dilated, and skeletonized. As a result, the final skeletal image was ready for FA. Finally, FD was measured using the ImageJ program with a box-counting algorithm (264 pixels).(Fig. 2)

The bone density measurements were based on the arrangement of trabeculae on cross-sectional images of $\mathrm{CBCT}^{27}$. Hence, GSVs of CBCT images were evaluated on crosssectional images, which were created from the panoramic format to avoid including the cortical bone. Cross-sectional images of CBCT with $1 \mathrm{~mm}$ thickness at $0.08 \mathrm{~mm}$ intervals were reconstructed. Subsequently, in every image, a square ROI $4 \mathrm{~mm}^{2}$ in area was set in the cancellous bone of the implant apex. As a result, the GSV at the implant apex of the ROI was measured with CBCT machine software.(Fig. 3) The measurements were calculated twice at a three-week interval by the same oral and maxillofacial radiologist to ensure intra-observer agreement. All initial FD values of mesial, distal, and apical sides and GSVs of apical sides were compared with the values obtained at the 12-month postoperative evaluation of functional loading of the implant.

\section{Statistical analysis}

We analyzed raw data using IBM SPSS Statistics software (ver. 21.0; IBM, Armonk, NY, USA). The methodology was reviewed by an independent statistician. Descriptive statistics were calculated and the Kolmogorov-Smirnov test was used to ensure normality. Since the data were not homogeneously distributed, non-parametric tests were used. Differences in DPR and CBCT FD values and GSVs immediately after implant surgery and at the 12-month postoperative evaluation of the functional loading of the implants were assessed using the Wilcoxon test. Spearman's test was used to assess correlations among GSV and FD measurements between the two specified periods. Values less than 0.05 were considered significant. The intra-observer agreement reliability was assessed by calculating Cronbach's alpha coefficients.

A power analysis software program was used for post hoc power calculation for data regarding FD. For $n=60$, group number 1 and $\alpha=0.05$ calculated power value for 0.5 effect size is $100 \%$.

\section{Results}

We included 16 patients (10 females, 6 males; mean age, $56 \pm 10$ years) with a total of 60 implants in the posterior mandibular area. All implants in this study were of the same brand (OsseoSpeed) with diameters of $4.0 \mathrm{~mm}$ and lengths of $11 \mathrm{~mm}$. All implants functioned successfully and no com-

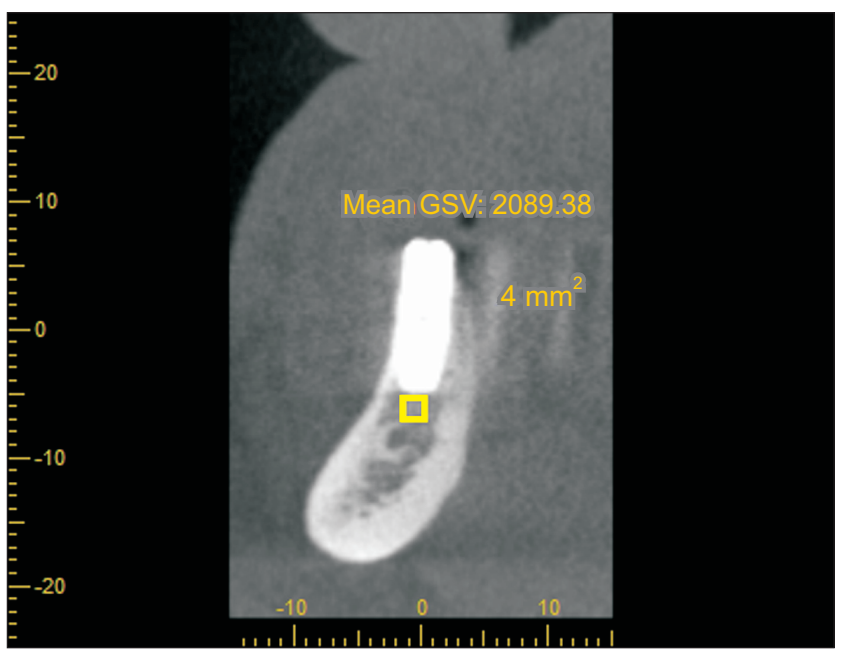

Fig. 3. The selection of grayscale value (GSV) $\left(4 \mathrm{~mm}^{2}\right)$ at the implant apex on the cross sectional image of cone-beam computed tomography (CBCT). The mean GSV was measured automatically by the CBCT software program.

Guldane Magat et al: Comparison of cone-beam computed tomography and digital panoramic radiography for detecting peri-implant alveolar bone changes using trabecular micro-structure analysis. J Korean Assoc Oral Maxillofac Surg 2022
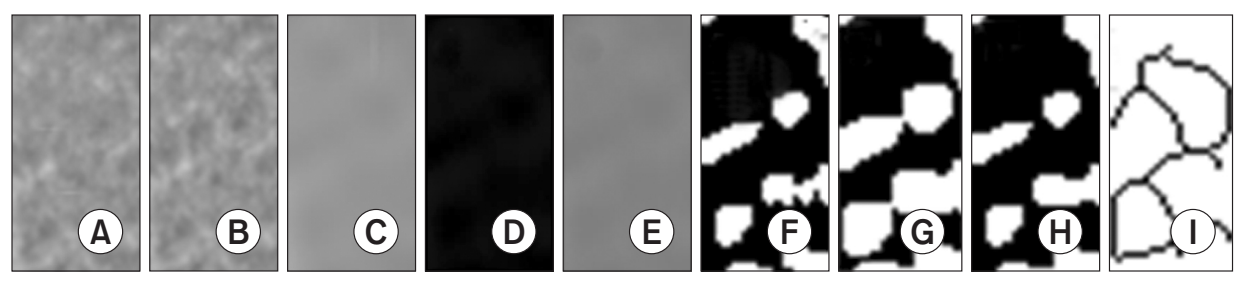

Fig. 2. A. Region of interest (ROI) on digital panoramic radiography and cone-beam volumetric tomography images were cropped and transferred to ImageJ. B, C. The cropped ROI was duplicated (B) and then blurred with a Gaussian filter (C). D, E. The blurred image was subtracted from the original image (D), and 128 was added to the result at each pixel location (E). F. The resultant image was converted to binary, to set the image into trabeculae and marrow spaces. G, H. The binary image was eroded then dilated to reduce the noise before skeletonization. I. The skeletonized image was used for fractal analysis.

Guldane Magat et al: Comparison of cone-beam computed tomography and digital panoramic radiography for detecting peri-implant alveolar bone changes using trabecular microstructure analysis. J Korean Assoc Oral Maxillofac Surg 2022 
plications developed during the surgery. The subjects did not complain of pain or have signs of inflammation in the region surrounding the implants. The soft tissues around implants were evaluated with a periodontal probe, and no inflammation was noted. At 12 weeks after surgery, the implants were assembled with porcelain-fused-to-metal cemented crowns.

The mean and standard deviation of FD values and GSVs for implants calculated right after implant surgery and at the

Table 1. FD values and GSVs after implant surgery and 12 months postoperative on DPR and reformatted panoramic images of CBCT $(\mathrm{n}=60)$

\begin{tabular}{clc}
\hline Timing & & Mean \pm SD \\
\hline After implant surgery & DPR distal FD & $1.17 \pm 0.09$ \\
& DPR apical FD & $1.14 \pm 0.09$ \\
& DPR mesial FD & $1.14 \pm 0.09$ \\
& CBCT distal FD & $1.18 \pm 0.07$ \\
& CBCT apical FD & $1.16 \pm 0.08$ \\
& CBCT mesial FD & $1.15 \pm 0.07$ \\
& Apical GSV & $2,118.53 \pm 366.00$ \\
12 months postoperative & DPR distal FD & $1.17 \pm 0.08$ \\
& DPR apical FD & $1.15 \pm 0.07$ \\
& DPR mesial FD & $1.15 \pm 0.08$ \\
& CBCT distal FD & $1.18 \pm 0.06$ \\
& CBCT apical FD & $1.15 \pm 0.07$ \\
& CBCT mesial FD & $1.16 \pm 0.07$ \\
& Apical GSV & $2,206.05 \pm 292.93$ \\
\hline
\end{tabular}

(FD: fractal dimension, GSVs: grayscale values, DPR: digital panoramic radiography, $\mathrm{CBCT}$ : cone-beam computed tomography, SD: standard deviation)

Guldane Magat et al: Comparison of cone-beam computed tomography and digital panoramic radiography for detecting peri-implant alveolar bone changes using trabecular micro-structure analysis. J Korean Assoc Oral Maxillofac Surg 2022 12-month postoperative evaluation of functional loading of the implant on DPR and CBCT images are displayed in Table 1.

The Cronbach's alpha values for intra-observer reliability ranged from 0.81 to 0.95 . No significant differences were found between FD values obtained from DPR at the distal, apical, and mesial sides measured right after implant surgery and at the 12-month postoperative visit, or between FD values obtained from $\mathrm{CBCT}$ at the distal, apical and mesial sides calculated during the two specified periods $(P>0.05)$. (Table 2) There were no significant differences between GSVs obtained from $\mathrm{CBCT}$ at the implant apex right after implant surgery and at the 12-month postoperative visit. There were no significant differences between FD values obtained from DPR and CBCT at the distal, apical, and mesial sides for both initial and final measurements $(P>0.05)$.(Table 3) According to Spearman's correlation tests, there was a significant correlation between FD values obtained from DPR and CBCT at the distal, apical, and mesial sides assessed right after the implant surgery and at the 12 -month postoperative visit. However, GSVs at the implant apex were not correlated with the FD values obtained from DPR and CBCT at the distal, apical, and mesial sides after implant surgery and at the 12-month postoperative visit $(P>0.05)$.

Table 2. Comparison of FD values after implant surgery and at the 12-month postoperative (postop) visit on DPR and reformatted panoramic images of CBCT

\begin{tabular}{lc}
\hline \multicolumn{1}{c}{ Variable } & $P$-value \\
\hline DPR distal FD after implant surgery to DPR distal FD at the 12-month postop & 0.520 \\
DPR apical FD after implant surgery to DPR apical FD at the 12-month postop & 0.895 \\
DPR mesial FD after implant surgery to DPR mesial FD at the 12-month postop & 0.639 \\
CBCT distal FD after implant surgery to DPR distal FD at the 12-month postop & 0.560 \\
CBCT apical after implant surgery to DPR apical FD at the 12-month postop & 0.444 \\
CBCT mesial FD after implant surgery to DPR mesial FD at the 12-month postop & 0.567 \\
Apical GSV after implant surgery to apical GSV at the 12-month postop & 0.242
\end{tabular}

(FD: fractal dimension, DPR: digital panoramic radiography, CBCT: cone-beam computed tomography, GSV: grayscale value)

Guldane Magat et al: Comparison of cone-beam computed tomography and digital panoramic radiography for detecting peri-implant alveolar bone changes using trabecular microstructure analysis. J Korean Assoc Oral Maxillofac Surg 2022

Table 3. $P$-values between the FD values obtained from DPR and reformatted panoramic images of CBCT

\begin{tabular}{lc}
\hline & Variable \\
\hline DPR distal FD after implant surgery to CBCT distal FD after implant surgery & 0.178 \\
DPR apical FD after implant surgery to CBCT apical FD after implant surgery & 0.460 \\
DPR mesial FD after implant surgery to CBCT mesial FD after implant surgery & 0.271 \\
DPR distal FD at the 12-month postop to CBCT distal FD at the 12-month postop & 0.140 \\
DPR apical FD at the 12-month postop to CBCT apical FD at the 12-month postop & 0.196 \\
DPR mesial FD at the 12-month postop to CBCT mesial FD at the 12-month postop & 0.195 \\
\hline
\end{tabular}

(FD: fractal dimension, DPR: digital panoramic radiography, CBCT: cone-beam computed tomography, postop: postoperative)

Guldane Magat et al: Comparison of cone-beam computed tomography and digital panoramic radiography for detecting peri-implant alveolar bone changes using trabecular microstructure analysis. J Korean Assoc Oral Maxillofac Surg 2022 


\section{Discussion}

We compared changes in trabecular bone architecture measured on radiological images obtained after implant surgery and at 12 months postoperative. This study is the first evaluating FD and GSV of peri-implant alveolar bone on DPRs and CBCTs immediately after implant surgery and at the 12-month postoperative visit, and the relationships between FD values on DPR and CBCT.

All measurements were assessed on DPR and CBCT images. A dentomaxillofacial radiologist (G.M.) evaluated each image to ensure reliability and consistency. The Cronbach's alpha values were excellent, demonstrating that the methods used for evaluation were dependable. All implants were placed by the same surgeon (E.O.) using the same technique and identical brands and implant sizes, to reduce experimental variation to a minimum.

Koh et al. ${ }^{31}$ determined that the mandibular premolar-molar area was the best site to assess FD values from DPR images, while the posterior mandible was more suitable for mechanical power and to display noticeable changes in the trabecular bone $^{32}$. Therefore, we assessed implants in the posterior mandible in this study.

Trabecular bone evaluation has been a dependable standout among the most critical parameters for foreseeing long-term success in dental implant therapy. In this study, we analyzed trabecular patterns on DPR and CBCT images using FD measurements as well as correlations between GSV and FD values obtained from DPR and $\mathrm{CBCT}$ associated with bone quality.

Trabecular bone has a branching pattern that displays fractal properties such as self-similitude and the absence of a well-defined scale. Fractal geometry and FD measurements can be used to detect trabecular complexity and bone structure $^{33}$. FA of the bone trabecular structure is suitable evaluating bone quality in clinical applications ${ }^{34}$.

FD is a mathematical method to measure complex structures. Generally, more complex shapes possess higher FD Southard et al. ${ }^{35}$ detected a positive relationship between bone density and FD measurements. As bone density increases, FD increases. In this study, FA was performed on DPR and $\mathrm{CBCT}$ images for the noninvasive assessment of trabecular bone structures to compare FD values between DPR and CBCT.

FD values of healthy bone ranged from 1.1 to $2.68^{16,35,36}$. This instability was predominantly caused by the issues of the FA itself rather than by various materials and strategies, anatomic areas, and ROI estimates used in every study. In the current study, FD values of DPR and CBCT for implant sites were $1.17 \pm 0.09,1.14 \pm 0.09,1.14 \pm 0.09,1.17 \pm 0.08$, $1.15 \pm 0.07,1.15 \pm 0.08,1.18 \pm 0.07,1.16 \pm 0.08,1.15 \pm 0.07$, $1.18 \pm 0.06,1.15 \pm 0.07$, and $1.16 \pm 0.07$ for distal, apical, and mesial areas, respectively, in alignment with values reported in the literature.

Very few studies have compared panoramic radiography and CBCT-related image quality for assessment of $\mathrm{FD}^{37-39}$. In previous studies, conventional panoramic radiographs had much higher spatial resolution than either CBCTs or medical CT due to image blurring. In this study, there were no significant differences between FD values obtained from DPR and CBCT $(P>0.05)$. Hence, DPR and reconstructed panoramic images of CBCT had similar image qualities for the assessment of FD. On the other hand, no study has compared DPRs with CBCT using the FA for image quality. The present study was the first to clinically compare FD values obtained from DPR and CBCT in jaw bone morphometry.

The trabecular bone surrounding dental implants has been assessed occasionally in the past by $\mathrm{FA}^{13,34,40-42}$. In recent studies by dos Santos Corpas et al. ${ }^{40}$ and Ilhan et al. ${ }^{42}$, no changes in peri-implant bone tissue were found after loading. Meanwhile, some studies have found that there was a significant increase in mean FD value after prosthodontic loading ${ }^{34,41}$. Zeytinoğlu et al. ${ }^{13}$ however found that peri-implant alveolar bone did not significantly decrease or increase at 12 months post-implantation compared to 6 months post-implantation, similar to our results.

There are fewer studies considering the FD on CBCT. Hua et al. ${ }^{43}$ stated that the drop in CBCT FD values was significantly correlated with reduction of bone density as measured with dual-energy X-ray absorptiometry. GonzálezMartín et al. ${ }^{44}$ found that the mean FD value at the implant apex displayed a $3 \%$ increase in the 6 months following the emplacement. Huang et al. ${ }^{45}$ reported that FD results did not differ due to bone remodeling. In this study, analysis of the changes in peri-implant trabecular bone architecture did not reveal any significant differences immediately after implant surgery and at the 12-month follow-up. This result is consistent with previous studies conducted by Jung ${ }^{17}$, Ilhan et al. ${ }^{42}$, and Zeytinoğlu et al. ${ }^{13}$. The differences between the results may also reflect the influence of physiological functions and mechanical loading on the trabecular bone architecture ${ }^{11}$. Related research showed that all implants lead to increases of bone density in the apical side, but the reason conventionally loaded implants demonstrate this pattern remains unclear ${ }^{46,47}$. 
The periapical bone surrounding the implants may be exposed to chewing stress over a longer time than implants that are loaded continuously. By avoiding early overwhelming stresses, the crestal bone around the implant that is loaded continuously becomes able to support the overloading capacity and reduce the amount of load to the apical bone around the implant. This slight stimulation may produce bone in this peri-implant region ${ }^{27}$.

Studies based on bone density analysis of grayscale CBCT images are controversial. Several studies have reported high correlations between the intensity values of $\mathrm{CBCT}$ and $\mathrm{HU}$ of multi-slice $\mathrm{CT}^{24,26}$. Some researchers reported significant correlations between bone density and implant stability ${ }^{21}$. Throughout in vitro studies ${ }^{26}$, grayscale intensity values quantitatively measured in CBCT groups (GSV) were generally found to be higher than those obtained from the CT groups (HU). Technical factors such as X-ray beam hardening, scatter radiation, and the "discontinuity-related effect of projection data" are said to cause this discrepancy. These are all affected by the contrast of the dynamic differentiation of CBCT scanners (8-14 bits) with multi-slice CT (16 bits) ${ }^{48}$. Along with increased radio-opacity (mineral content), radiation hardening becomes more noticeable ${ }^{49}$. Clinicians should be aware of this fact when making clinical decisions. In the present study, the mean GSVs at the implant apex after implant surgery and at the 12-month postoperative visit were $2,118.53 \pm 366.00$ and $2,206.05 \pm 292.93$, respectively. There were no significant correlations with the GSVs obtained from CBCT and FD values obtained from DPR and CBCT after implant surgery and at the 12-month postoperative visit. No significant differences were observed between GSVs at the implant apex after implant surgery and at the 12-month postoperative visit.

The outcomes of this study demonstrate that loading stress did not increase FD values and GSVs. Three important factors that affected bone-related responses to loaded dental implants were mechanical effects, implant design, and implant surface characteristics ${ }^{16}$. All of the implant fixtures (OsseoSpeed) used in the present study had the same surface treatment, implant-abutment interface (conical seal design; Astra Tech), and thread characteristics. We confirmed that trabecular bone displays directional anisotropy of its mechanical components and design, dependent on its physiological function and mechanical loading over the skeleton ${ }^{50}$. More research is needed to fully standardize image processing methods, minimize artifacts, and optimize the reconstruction algorithm for practical clinical applications of $\mathrm{CBCT}$ in peri- implant bone tissue analyses ${ }^{45}$.

There are a few limitations to this study. First, this study concentrated on recognizing changes in trabecular architecture on clinical digital images using FA. No comparisons were performed with other diagnostic techniques. FD, which is obtained from CT images and micro-CT, has greater potential to interpret the microarchitecture of bone and facilitate the whole characterization of trabecular mesh than other modalities ${ }^{43}$. Although using both radiographic techniques increases radiation exposure, this can be reduced to a minimum by careful choice of exposure parameters. Analysis of 3D images may be more popular than 2D images in the future. Second, we used a short follow-up period, but the amount of peri-implant trabecular bone increases over a period of longer than one year ${ }^{13}$. Additional long-term studies are required to assess the relationships between FD changes and functional loading. Third, we used a combined surgical procedure with a single type of implant design. Additional studies are required to determine the impacts of various dental implant systems on trabecular bone structure. Finally, we included a single CBCT model. It has been demonstrated that there is significant inconsistency in spatial and contrast resolution between CBCTs, with the model used in this study to be a leading choice on the image quality range scale ${ }^{27}$. Therefore, no conclusions can be made regarding $\mathrm{CBCT}$ as a whole as noted above, and differences between CBCTs as a result of varying exposure and reconstruction factors should be further investigated. The GSVs in this study should not be applied to other CBCT devices until directed by further predictive studies.

\section{Conclusion}

This study revealed that DPR and CBCT are useful for assessing trabecular bone design. The DPR and reconstructed panoramic images of $\mathrm{CBCT}$ have similar image quality for the assessment of FD. The FD values and GSVs of the periimplant trabecular bone architecture did not show changes at the 12-month postoperative evaluations of functional loading of the implants, and the FD values obtained from DPR and CBCT immediately after implant surgery and at the 12-month postoperative visit were not correlated with the GSV. Therefore, GSVs representing bone mass do not support FD values that predict bone microstructure. We recommend that these measures should be considered different parameters for assessing bone quality. The use of $\mathrm{CBCT}$ for FD analysis would contribute towards avoiding the superimposition of cortical bone on trabecular bone, which would give rise to more pre- 
cise FD values. However, considering the radiation dose, image resolution, and expenses of $\mathrm{CBCT}$ machines, it is more appropriate to use DPR instead of CBCT. FD is applicable and makes clinical sense in implantology. More detailed studies are needed to confirm these outcomes and to test their clinical appropriateness.

\section{ORCID}

Guldane Magat, https://orcid.org/0000-0003-4418-174X

Elif Oncu, https://orcid.org/0000-0002-5774-4604

Sevgi Ozcan, https://orcid.org/0000-0002-2349-9292

Kaan Orhan, https://orcid.org/0000-0001-6768-0176

\section{Authors' Contributions}

G.M. and E.O. made substantial contributions to conception and design, and/or acquisition of data, and/or analysis and interpretation of data. S.O. and K.O. participated in drafting the article or revising it critically for important intellectual content. All authors gave final approval of the version to be submitted and any revised version.

\section{Acknowledgements}

This research was conducted at the Necmettin Erbakan University, Faculty of Dentistry, Konya, Turkey. The authors thank Mr. Gokhan Kocturk (https://tr.linkedin.com/in/ gökhan-koçtürk) for statistician review.

\section{Ethics Approval and Consent to Participate}

This retrospective study was approved by the Local Research Ethics Committee (No. 2018.01) and the informed consent was waived by the Research Ethics Committee.

\section{Conflict of Interest}

No potential conflict of interest relevant to this article was reported.

\section{References}

1. Donos N, Mardas N, Chadha V. Clinical outcomes of implants following lateral bone augmentation: systematic assessment of available options (barrier membranes, bone grafts, split osteotomy). J Clin Periodontol 2008;35(8 Suppl):173-202. https://doi. org/10.1111/j.1600-051X.2008.01269.x
2. Retzepi M, Donos N. Guided bone regeneration: biological principle and therapeutic applications. Clin Oral Implants Res 2010;21: 567-76. https://doi.org/10.1111/j.1600-0501.2010.01922.x

3. Santing HJ, Raghoebar GM, Vissink A, den Hartog L, Meijer HJ. Performance of the Straumann Bone Level Implant system for anterior single-tooth replacements in augmented and nonaugmented sites: a prospective cohort study with 60 consecutive patients. Clin Oral Implants Res 2013;24:941-8. https://doi.org/10.1111/j.16000501.2012.02486.x

4. Shalabi MM, Wolke JG, Jansen JA. The effects of implant surface roughness and surgical technique on implant fixation in an in vitro model. Clin Oral Implants Res 2006;17:172-8. https://doi. org/10.1111/j.1600-0501.2005.01202.x

5. Brunski JB, Puleo DA, Nanci A. Biomaterials and biomechanics of oral and maxillofacial implants: current status and future developments. Int J Oral Maxillofac Implants 2000;15:15-46.

6. Stanford CM, Brand RA. Toward an understanding of implant occlusion and strain adaptive bone modeling and remodeling. J Prosthet Dent 1999;81:553-61. https://doi.org/10.1016/s00223913(99)70209-x

7. Bidez MW, Misch CE. Force transfer in implant dentistry: basic concepts and principles. J Oral Implantol 1992;18:264-74.

8. Drago CJ. Rates of osseointegration of dental implants with regard to anatomical location. J Prosthodont 1992;1:29-31. https://doi. org/10.1111/j.1532-849x.1992.tb00423.x

9. Jemt T, Lekholm U. Oral implant treatment in posterior partially edentulous jaws: a 5-year follow-up report. Int J Oral Maxillofac Implants 1993;8:635-40.

10. Lee DH, Ku Y, Rhyu IC, Hong JU, Lee CW, Heo MS, et al. A clinical study of alveolar bone quality using the fractal dimension and the implant stability quotient. J Periodontal Implant Sci 2010;40:19-24. https://doi.org/10.5051/jpis.2010.40.1.19

11. Yasar F, Akgünlü F. Fractal dimension and lacunarity analysis of dental radiographs. Dentomaxillofac Radiol 2005;34:261-7. https:// doi.org/10.1259/dmfr/85149245

12. Huh KH, Baik JS, Yi WJ, Heo MS, Lee SS, Choi SC, et al. Fractal analysis of mandibular trabecular bone: optimal tile sizes for the tile counting method. Imaging Sci Dent 2011;41:71-8. https://doi. org/10.5624/isd.2011.41.2.71

13. Zeytinoğlu M, İlhan B, Dündar N, Boyacioğlu H. Fractal analysis for the assessment of trabecular peri-implant alveolar bone using panoramic radiographs. Clin Oral Investig 2015;19:519-24. https:// doi.org/10.1007/s00784-014-1245-y

14. Jolley L, Majumdar S, Kapila S. Technical factors in fractal analysis of periapical radiographs. Dentomaxillofac Radiol 2006;35:3937. https://doi.org/10.1259/dmfr/30969642

15. Melsen B, Lang NP. Biological reactions of alveolar bone to orthodontic loading of oral implants. Clin Oral Implants Res 2001;12:144-52. https://doi.org/10.1034/j.16000501.2001.012002144.x

16. Bianchi AE, Dolci G Jr, Sberna MT, Sanfilippo S. Factors affecting bone response around loaded titanium dental implants: a literature review. J Appl Biomater Biomech 2005;3:135-40.

17. Jung YH. Evaluation of peri-implant bone using fractal analysis. Korean J Oral Maxillofac Radiol 2005;35:121-5.

18. Perrotti V, Aprile G, Degidi M, Piattelli A, Iezzi G. Fractal analysis: a novel method to assess roughness organization of implant surface topography. Int J Periodontics Restorative Dent 2011;31:633-9.

19. Bollen AM, Taguchi A, Hujoel PP, Hollender LG. Fractal dimension on dental radiographs. Dentomaxillofac Radiol 2001;30:2705. https://doi.org/10.1038/sj/dmfr/4600630

20. Dohan Ehrenfest DM. Fractal patterns applied to implant surface: definitions and perspectives. J Oral Implantol 2011;37:506-9. https://doi.org/10.1563/AAID-JOI-D-11-00081

21. González-García R, Monje F. Is micro-computed tomography reliable to determine the microstructure of the maxillary alveolar bone? Clin Oral Implants Res 2013;24:730-7. https://doi. 
org/10.1111/j.1600-0501.2012.02478.x

22. Müller R, Van Campenhout H, Van Damme B, Van Der Perre G, Dequeker J, Hildebrand T, et al. Morphometric analysis of human bone biopsies: a quantitative structural comparison of histological sections and micro-computed tomography. Bone 1998;23:59-66. https://doi.org/10.1016/s8756-3282(98)00068-4

23. Swain MV, Xue J. State of the art of Micro-CT applications in dental research. Int J Oral Sci 2009;1:177-88. https://doi.org/10.4248/ IJOS09031

24. Aranyarachkul P, Caruso J, Gantes B, Schulz E, Riggs M, Dus I, et al. Bone density assessments of dental implant sites: 2. Quantitative cone-beam computerized tomography. Int J Oral Maxillofac Implants 2005;20:416-24.

25. Turkyilmaz I, Sennerby L, McGlumphy EA, Tözüm TF. Biomechanical aspects of primary implant stability: a human cadaver study. Clin Implant Dent Relat Res 2009;11:113-9. https://doi. org/10.1111/j.1708-8208.2008.00097.x

26. Naitoh M, Hirukawa A, Katsumata A, Ariji E. Evaluation of voxel values in mandibular cancellous bone: relationship between conebeam computed tomography and multislice helical computed tomography. Clin Oral Implants Res 2009;20:503-6. https://doi. org/10.1111/j.1600-0501.2008.01672.x

27. Pauwels R, Nackaerts O, Bellaiche N, Stamatakis H, Tsiklakis K, Walker A, et al.; SEDENTEXCT Project Consortium. Variability of dental cone beam CT grey values for density estimations. Br J Radiol 2013;86:20120135. https://doi.org/10.1259/bjr.20120135

28. Vandenberghe B, Jacobs R, Bosmans H. Modern dental imaging: a review of the current technology and clinical applications in dental practice. Eur Radiol 2010;20:2637-55. https://doi.org/10.1007/ s00330-010-1836-1

29. Tyndall DA, Price JB, Tetradis S, Ganz SD, Hildebolt C, Scarfe WC; American Academy of Oral and Maxillofacial Radiology. Position statement of the American Academy of Oral and Maxillofacial Radiology on selection criteria for the use of radiology in dental implantology with emphasis on cone beam computed tomography. Oral Surg Oral Med Oral Pathol Oral Radiol 2012;113: 817-26. https://doi.org/10.1016/j.oooo.2012.03.005

30. White SC, Cohen JM, Mourshed FA. Digital analysis of trabecular pattern in jaws of patients with sickle cell anemia. Dentomaxillofac Radiol 2000;29:119-24. https://doi.org/10.1038/sj/dmfr/4600516

31. Koh KJ, Park HN, Kim KA. Prediction of age-related osteoporosis using fractal analysis on panoramic radiographs. Imaging Sci Dent 2012;42:231-5. https://doi.org/10.5624/isd.2012.42.4.231

32. Brånemark PI, Hansson BO, Adell R, Breine U, Lindström J, Hallén $\mathrm{O}$, et al. Osseointegrated implants in the treatment of the edentulous jaw. Experience from a 10-year period. Scand J Plast Reconstr Surg Suppl 1977;16:1-132.

33. Haire TJ, Hodgskinson R, Ganney PS, Langton CM. A comparison of porosity, fabric and fractal dimension as predictors of the Young's modulus of equine cancellous bone. Med Eng Phys 1998; 20:588-93. https://doi.org/10.1016/s1350-4533(98)00063-0

34. Wilding RJ, Slabbert JC, Kathree H, Owen CP, Crombie K, Delport P. The use of fractal analysis to reveal remodelling in human alveolar bone following the placement of dental implants. Arch Oral Biol 1995;40:61-72. https://doi.org/10.1016/0003-9969(94)001382

35. Southard TE, Southard KA, Jakobsen JR, Hillis SL, Najim CA. Fractal dimension in radiographic analysis of alveolar process bone. Oral Surg Oral Med Oral Pathol Oral Radiol Endod 1996;82: 569-76. https://doi.org/10.1016/s1079-2104(96)80205-8

36. Geraets WG, van der Stelt PF. Fractal properties of bone. Dentomaxillofac Radiol 2000;29:144-53. https://doi.org/10.1038/sj/ $\mathrm{dmfr} / 4600524$

37. Pawelzik J, Cohnen M, Willers R, Becker J. A comparison of conventional panoramic radiographs with volumetric computed tomography images in the preoperative assessment of impacted mandibular third molars. J Oral Maxillofac Surg 2002;60:979-84. https://doi.org/10.1053/joms.2002.34399

38. Mischkowski RA, Ritter L, Neugebauer J, Dreiseidler T, Keeve E, Zöller JE. Diagnostic quality of panoramic views obtained by a newly developed digital volume tomography device for maxillofacial imaging. Quintessence Int 2007;38:763-72.

39. Pittayapat P, Galiti D, Huang Y, Dreesen K, Schreurs M, Souza PC, et al. An in vitro comparison of subjective image quality of panoramic views acquired via $2 \mathrm{D}$ or $3 \mathrm{D}$ imaging. Clin Oral Investig 2013;17:293-300. https://doi.org/10.1007/s00784-012-0698-0

40. dos Santos Corpas L, Jacobs R, Quirynen M, Huang Y, Naert I, Duyck J. Peri-implant bone tissue assessment by comparing the outcome of intra-oral radiograph and cone beam computed tomography analyses to the histological standard. Clin Oral Implants Res 2011;22:492-9. https://doi.org/10.1111/j.1600-0501.2010.02029.x

41. Sansare K, Singh D, Karjodkar F. Changes in the fractal dimension on pre- and post-implant panoramic radiographs. Oral Radiol 2012; 28:15-23. https://doi.org/10.1007/s11282-011-0075-8

42. Ilhan B, Güneri P, Saraçoglu A, Koca H, Boyacioglu H. A comparison of fractal dimension values of peri-implant bone and healthy contralateral side using panoramic radiographs. J Oral Maxillofac Radiol 2015;3:1-6. https://doi.org/10.4103/2321-3841.151636

43. Hua Y, Nackaerts O, Duyck J, Maes F, Jacobs R. Bone quality assessment based on cone beam computed tomography imaging. Clin Oral Implants Res 2009;20:767-71. https://doi.org/10.1111/j.16000501.2008.01677.x

44. González-Martín O, Lee EA, Veltri M. CBCT fractal dimension changes at the apex of immediate implants placed using undersized drilling. Clin Oral Implants Res 2012;23:954-7. https://doi. org/10.1111/j.1600-0501.2011.02246.x

45. Huang Y, Van Dessel J, Liang X, Depypere M, Zhong W, Ma G, et al. Effects of immediate and delayed loading on peri-implant trabecular structures: a cone beam CT evaluation. Clin Implant Dent Relat Res 2014;16:873-83. https://doi.org/10.1111/cid.12063

46. Appleton RS, Nummikoski PV, Pigno MA, Cronin RJ, Chung KH. A radiographic assessment of progressive loading on bone around single osseointegrated implants in the posterior maxilla. Clin Oral Implants Res 2005;16:161-7. https://doi.org/10.1111/j.16000501.2004.01089.x

47. Barone A, Covani U, Cornelini R, Gherlone E. Radiographic bone density around immediately loaded oral implants. Clin Oral Implants Res 2003;14:610-5. https://doi.org/10.1034/j.16000501.2003.00878.x

48. Goodsitt MM, Chan HP, Way TW, Larson SC, Christodoulou EG, Kim J. Accuracy of the CT numbers of simulated lung nodules imaged with multi-detector CT scanners. Med Phys 2006;33:3006-17. https://doi.org/10.1118/1.2219332

49. Mori S, Endo M, Tsunoo T, Kandatsu S, Tanada S, Aradate H, et al. Physical performance evaluation of a 256-slice CT-scanner for four-dimensional imaging. Med Phys 2004;31:1348-56. https://doi. org/10.1118/1.1747758

50. Yi WJ, Heo MS, Lee SS, Choi SC, Huh KH, Lee SP. Direct measurement of trabecular bone anisotropy using directional fractal dimension and principal axes of inertia. Oral Surg Oral Med Oral Pathol Oral Radiol Endod 2007;104:110-6. https://doi.org/10.1016/ j.tripleo.2006.11.005

How to cite this article: Magat G, Oncu E, Ozcan S, Orhan K. Comparison of cone-beam computed tomography and digital panoramic radiography for detecting peri-implant alveolar bone changes using trabecular micro-structure analysis. J Korean Assoc Oral Maxillofac Surg 2022;48:41-49. https://doi.org/10.5125/ jkaoms.2022.48.1.41 